\title{
Aplicativo móvel para avaliação, prevenção e tratamento da dermatite associada à incontinência
}

\author{
Mobile application for evaluation, \\ prevention and treatment of dermatitis \\ associated with incontinence
}

\section{Geraldo Magela Salomé1 1 (1) Cleber Aparecido da Rocha² (1)}

${ }^{1}$ Autor para correspondência. Universidade do Vale do Sapucaí (Pouso Alegre). Minas Gerais, Brasil. salomereiki@univas.edu.br ${ }^{2}$ Universidade do Vale do Sapucaí (Pouso Alegre). Minas Gerais, Brasil. cleber@blueorbis.org

\begin{abstract}
RESUMO | OBJETIVOS: Desenvolver um aplicativo para auxiliar na avaliação, prevenção e tratamento da dermatite associada à incontinência. MÉTODO: Estudo aplicado à produção tecnológica na área da saúde. Para o design do aplicativo, foram elaborados: conteúdo didático, definição dos itens relacionados à avaliação, prevenção e tratamento da dermatite associada à incontinência e foi feita a seleção das mídias. Foram escolhidos os dispositivos do aplicativo. Na fase de implementação, os dispositivos e os recursos tecnológicos foram instalados, e foi construído um ambiente para baixar o aplicativo na Internet e implementar a funcionalidade do aplicativo móvel. RESULTADOS: Após revisão integrativa da literatura, foi desenvolvido o DIAPERSKIN com 22 telas e 8 figuras. Ele foi registrado no Instituto Nacional da Propriedade Industrial sob o número BR-51-2018-000-720-1 e está disponível gratuitamente no Google Play Store. CONCLUSÃO: Foram descritos o planejamento e desenvolvimento, com base em evidências, de um aplicativo móvel para a avaliação, prevenção e tratamento de DAl, o qual poderá ser útil durante a assistência, na avaliação de pacientes, seleção de ações preventivas e na indicação do tratamento, assim como para a educação continuada de profissionais da enfermagem por meio do uso da tecnologia de fácil utilização.
\end{abstract}

DESCRITORES: Dermatite. Dermatite das Fraldas. Software. Aplicações da informática médica. Promoção da saúde.

\begin{abstract}
OBJECTIVE: To develop a mobile application to assist in the assessment, prevention and treatment of incontinence-associated dermatitis. METHODS: A study on the use of mobile technology in health care. The design of the mobile app involved the creation of didactic content, definition of topics related to the assessment, prevention, and treatment of IAD, selection of media, and interface design. In the implementation phase, tools and technological resources were configured, and an environment was built to download the app from the Internet and install it on a mobile device. RESULTS: Following an integrative review of the literature, the DIAPERSKIN mobile app was created containing 22 screens and 8 figures. It was registered with the Brazilian National Institute of Industrial Property (protocol number BR-51-2018-000-720-1) and is freely available in the Google Play Store. CONCLUSION: This study described the evidencebased planning and development of a mobile app for the assessment, prevention and treatment of IAD, which may be useful during clinical practice in the assessment of patients, selection of preventive measures and therapeutic approaches, and for continuing nursing education through an easy-to-use technology.
\end{abstract}

DESCRIPTORS: Dermatitis. Diaper Dermatitis. Computer software. Medical informatics applications. Health promotion. 


\section{Introdução}

Vários fatores ambientais relacionados ao envelhecimento afetam a hidratação da pele e a eficiência de sua função como barreira, aumentando o risco de dermatite associada à incontinência (DAI). A incidência de DAI é maior entre idosos, sendo ocasionada pela incontinência urinária e fecal ${ }^{1-4}$.

Define-se a DAI como uma área com presença de eritema e edema, podendo apresentar lesões bolhosas com exsudato, erosão ou infecção cutânea secundária. Estes sintomas devem-se à exposição constante dessa região à presença de efluentes, tais como urina, fezes, perspiração e exsudato da lesão. Também indivíduos jovens podem desenvolver DAl, principalmente aqueles que usam fraldas e são dependentes da equipe de Enfermagem para manter a higiene corporal ${ }^{5-8}$.

A DAl como uma injúria distinta tem motivado a realização, nos últimos anos, de pesquisas para a descrição de sua etiologia, diagnóstico e tratamento. Estima-se que acometa cerca de $30 \%$ dos idosos que vivem na comunidade, de $40 \%$ a $70 \%$ daqueles hospitalizados e $50 \%$ dos idosos que vivem em instituições de longa permanência para ${ }^{9-10}$.

A Enfermagem, atualmente, tem utilizado equipamentos tecnológicos como smartphones, tablets e notebooks, como meio de incrementar a sistematização da assistência em geral11-12. Estudo relata os benefícios e a rapidez para a prática clínica no uso de tais equipamentos, o que proporciona uma assistência individualizada, sistematizada, personalizada, como fator importante ao aprimoramento do cuidado ${ }^{12-16}$.

Os enfermeiros, por meio dessa tecnologia inovadora, têm acesso às informações acuradas que os auxiliam nas ações, garantindo o cuidado com qualidade e segurança. Os aplicativos móveis são facilmente transportados para hospitais e instituições de longa permanência, tanto urbanos como rurais, o que imprime maior destreza ao trabalho dos profissionais ${ }^{15-16}$.

O uso de aplicativos na prática clínica da Enfermagem contribui para a elaboração da assistência informatizada, facilita as atividades de coleta, anotação, armazenamento, manuseio e recuperação dos registros do indivíduo. Aplicativos possibilitam também a instrumentalização administrativa e auxiliam na tomada de decisões 13,17 .
Destaca-se a relevância de desenvolver um aplicativo móvel que ofereça ao enfermeiro um meio rápido de consulta para a prescrição de medidas preventivas e tratamento, como a limpeza da região perineal e perianal, com o objetivo de promover a cicatrização de lesão.

Esta pesquisa faz parte de um projeto de desenvolvimento de um aplicativo móvel com avatar para pessoas que apresentam fatores de risco para contraírem ou que já contraíram DAl. As seguintes perguntas foram feitas para orientar a construção do aplicativo: "Quais os estudos que existem na literatura sobre aplicativos móveis para prevenir e tratar esteticamente a DAl"? e "Quais diretrizes e recomendações foram propostas para o desenvolvimento desse aplicativo?"

O objetivo deste estudo foi desenvolver um aplicativo para auxiliar na avaliação, prevenção e tratamento da dermatite associada a incontinências.

\section{Métodos}

Estudo aplicado à produção tecnológica na área da saúde. O estudo foi aprovado pelo Comitê de Ética em Pesquisa da Faculdade de Ciências da Saúde Dr. José Antônio Garcia Coutinho (parecer $n^{\circ}$ CAAE 51545915.3.0000.5102) e conduzido entre janeiro a maio de 2018.

O aplicativo móvel para a avaliação, prevenção e tratamento da DAI foi desenvolvido em quatro etapas (Análise, Design, Desenvolvimento e Implementação), utilizando-se a metodologia de Design Instrucional Contextualizado $\frac{18}{}$, que envolve uma proposta construtivista e consiste na ação intencional de planejar, desenvolver e aplicar situações didáticas específicas, incorporando mecanismos que favoreçam a contextualização.

A etapa Análise consistiu em compreender o problema didático e resolver os problemas detectados.

Foi realizada uma revisão integrativa da literatura nas bases de dados: Biblioteca Cochrane, Scientific Electronic Library Online (SciELO), Literatura LatinoAmericana e do Caribe em Ciência da Saúde (LILACS), Sistema Online de Busca e Análise de Literatura 
Médica (MEDLINE), utilizando os descritores presentes em Ciência da Saúde/Medical Subject Headings DeSC/MeSH: pele; dermatite e dermatite das fraldas. A estratégia de busca para cada idioma foi determinada pela combinação dos descritores selecionados e o operador booleano "AND", conforme os exemplos: (1) pele AND dermatite; (2) pele $A N D$ dermatite $A N D$ dermatite de fralda. Também foi feita uma busca nas plataformas Google Play Store e Apple Store, com objetivo de detectar se havia aplicativo relacionado ao tema.

Os critérios de inclusão para a seleção das publicações foram: estudos primários que tivessem ligação direta com a temática, em português, inglês e espanhol, publicados nos anos de 2009 a 2019, e estarem disponíveis na íntegra. Foram excluídos capítulos de livros, teses, dissertações, monografias, relatórios técnicos e artigos que, após leitura do respectivo resumo, não convergiam com o objeto de estudo proposto, além das publicações que se repetiram nas bases de dados e biblioteca virtual. Também foram excluídos os artigos classificados com nível 6 (evidências baseadas em opiniões de especialistas).

Para classificar o nível de evidência dos estudos selecionados, foram observadas as categorias que abrangem seis níveis:

Nível 1: evidências resultantes da meta-análise de múltiplos ensaios clínicos controlados e randomizados;

Nível 2: evidências obtidas em estudos individuais com delineamento experimental;

Nível 3: evidências de estudos quase experimentais;

Nível 4: evidências de estudos descritivos (não experimentais) ou abordagem qualitativa;

Nível 5: evidências de relatos de caso ou experiência;

Nível 6: evidências baseadas em opiniões de especialistas.

Após a classificação do nível de evidências dos artigos selecionados na revisão interativa da literatura, foi construído um fluxograma descrevendo as etapas do aplicativo, o qual foi enviado para o programador de computação.

Fluxograma elaborado com base na literatura para auxiliar na construção do aplicativo, conforme a Figura 1 que segue.

Figura 1. Fluxograma para orientar a construção do aplicativo multimídia em plataforma móvel para a prevenção e o tratamento da dermatite associada à incontinência. Pouso Alegre, MG, Brasil, 2019

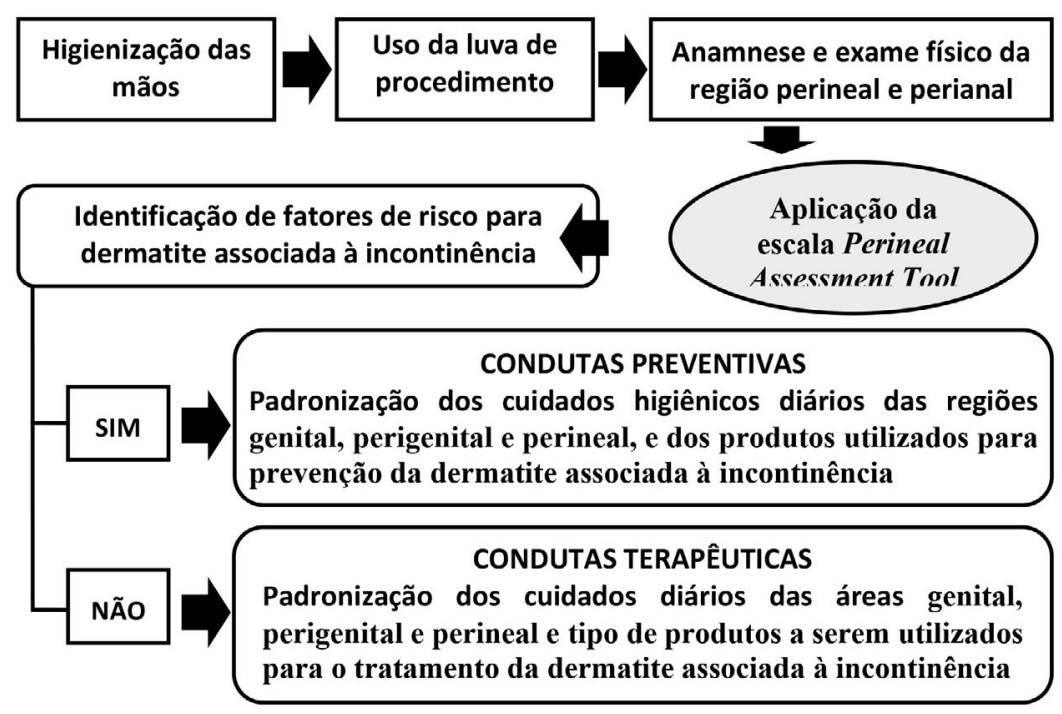


Os procedimentos a serem realizados na avaliação do paciente incluem: anamnese, exame físico com a avaliação da pele nas áreas genital, perigenital e perineal íntima, aplicação da escala Perineal Assessment Tool (PAT) ${ }^{19}$ versão brasileira e identificação dos fatores de risco para o indivíduo ser acometido por DAl. Seguem indicações dos cuidados e produtos a serem utilizados diariamente na higiene na região genital, perigenital e perineal íntima, e de medidas preventivas da DAI com base nos resultados do exame físico, anamnese e escores na escala PAT.

Foi feita a padronização de condutas terapêuticas para o tratamento da DAI com a sequência correta de procedimentos e a utilização de produtos adequados na higiene nas áreas genital, perigenital e perineal, com base nos resultados da avaliação dessas regiões e nos escores da escala PAT.

Foram criados vários desenhos que ilustram de maneira clara os procedimentos propostos, não só para o profissional que os indica, mas também para o paciente leigo que poderá optar por um ou outro método, quando encaminhado para o enfermeiro.

Na etapa Design, os pesquisadores efetuaram uma programação e a construção do conteúdo didático, escolha dos itens relacionados à avaliação, prevenção e tratamento da DAl e redação dos temas, seleção das mídias e da figura da interface (layout). Decidiu-se recorrer a figuras e textos estruturados em tópicos e conectados por hipertextos (links). Todo este processo foi conduzido pelos autores desta pesquisa e pela equipe de programação de sistema.
Em Desenvolvimento, foram feitos a escolha dos dispositivos do aplicativo, a definição da estrutura de navegação e o planejamento da configuração de cada tela do aplicativo.

$\mathrm{Na}$ etapa Implementação, foi realizada a configuração dos dispositivos e da tecnologia educacional, bem como o desenvolvimento do ambiente para baixar o aplicativo na Internet, as instruções para utilizá-lo e a forma da instalação no dispositivo móvel.

\section{Resultados}

O aplicativo móvel desenvolvido nesse estudo é uma tecnologia dura, por se tratar da produção de um software, e fornece ao profissional uma ferramenta para auxiliar na avaliação, diagnóstico, prevenção e tratamento da DAI. Ele foi registrado no Instituto Nacional da Propriedade Industrial (protocolo número BR-51-2018-000-720-1) e está disponível no Google Play Store sob o nome DIAPERSKIN.

A interface do aplicativo possui um banco de dados composto pelo cadastro do paciente, contendo dados pessoais e aspectos clínicos, e pelo cadastro da avaliação da DAl, incluindo o registro da avaliação da região perianal e perineal, tipo e quantidade do exsudato, tipo de tecido, presença de infecção, localização anatômica da lesão e dados obtidos com a escala PAT.

O aplicativo desenvolvido é composto por 22 telas contendo 8 figuras. Imagens capturadas mostrando exemplos das telas do aplicativo DIAPERSKIN são apresentadas nas Figuras 2 a 5 na sequência. 
Figura 2. Exemplos das telas do aplicativo. (a) Início das avaliações ou consulta às referências bibliográficas; (b) Avaliação de fatores de risco; (c) Escala de avaliação perineal. Pouso Alegre, MG, Brasil, 2019
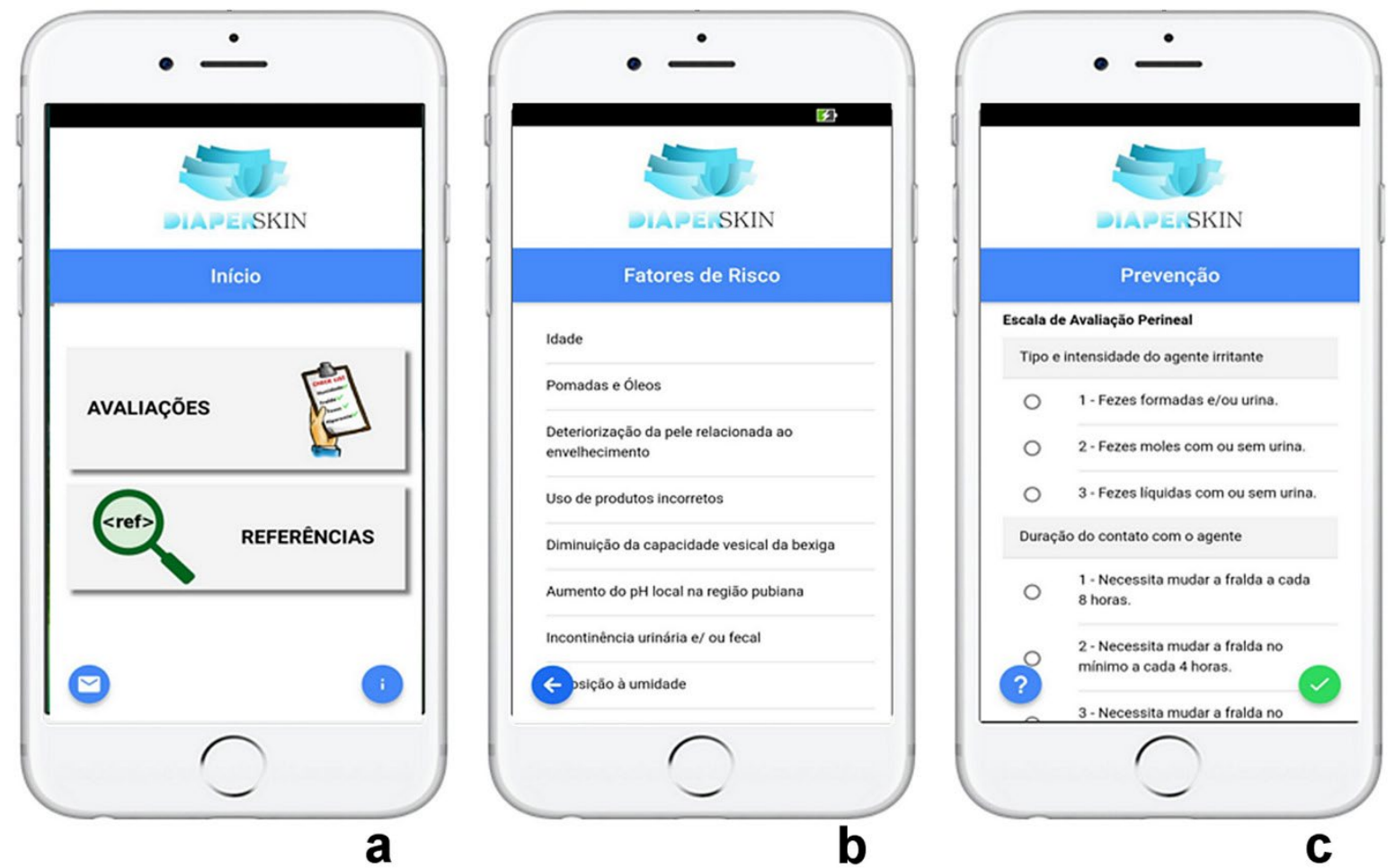

Figura 3. Exemplos das telas do aplicativo. (a) Opções de acesso a condutas preventivas ou de tratamento; (b) Higienização das mãos antes dos procedimentos; (c) Texto parcial sobre condutas terapêuticas preventivas. Pouso Alegre, MG, Brasil, 2019

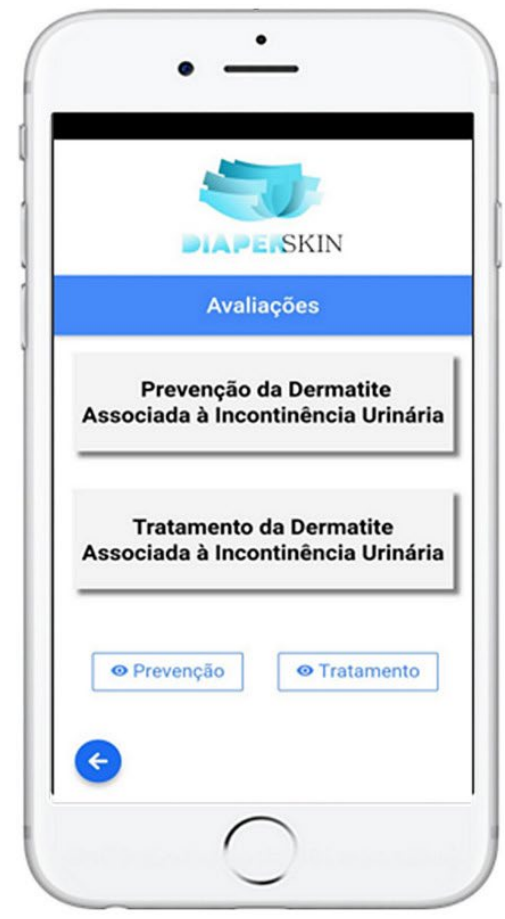

a

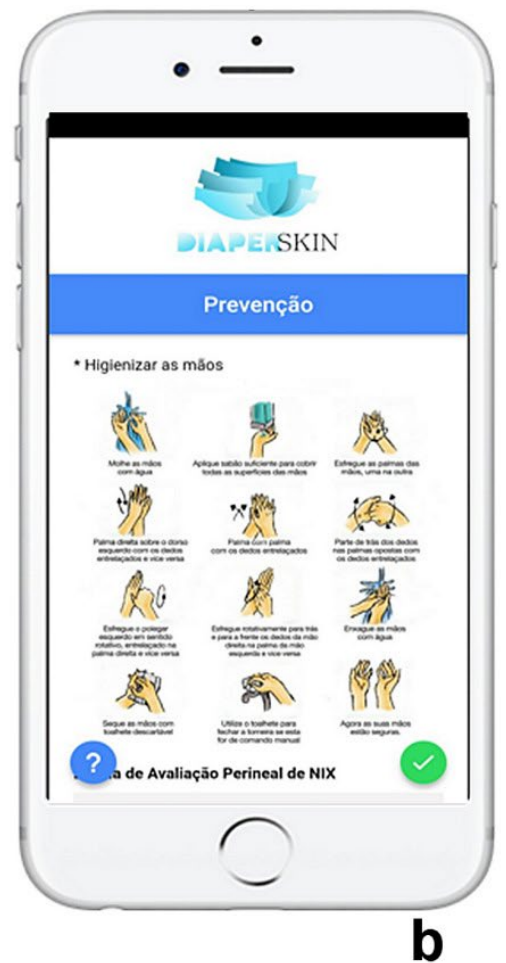


Figura 4. Exemplos de telas do aplicativo. (a) Tratamento preventivo; (b) Algoritmo do protocolo de prevenção; (c) Escala de avaliação da dermatite. Pouso Alegre, MG, Brasil, 2019

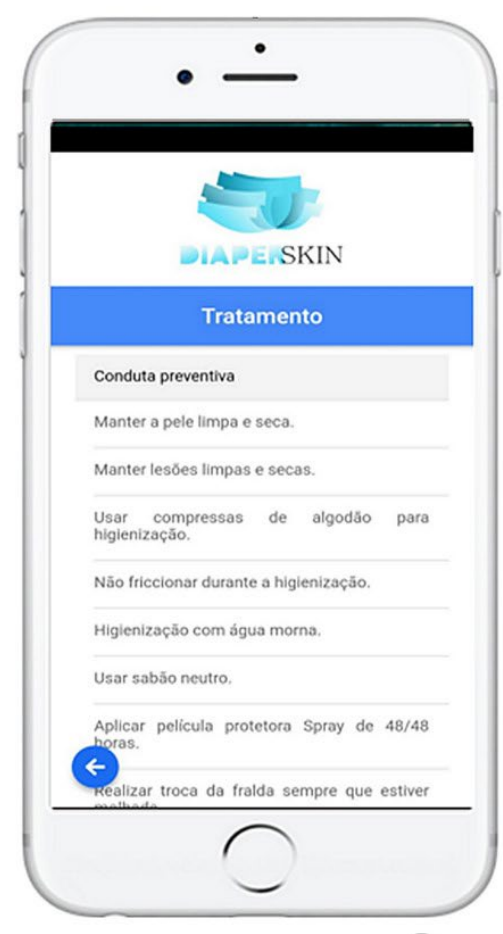

a

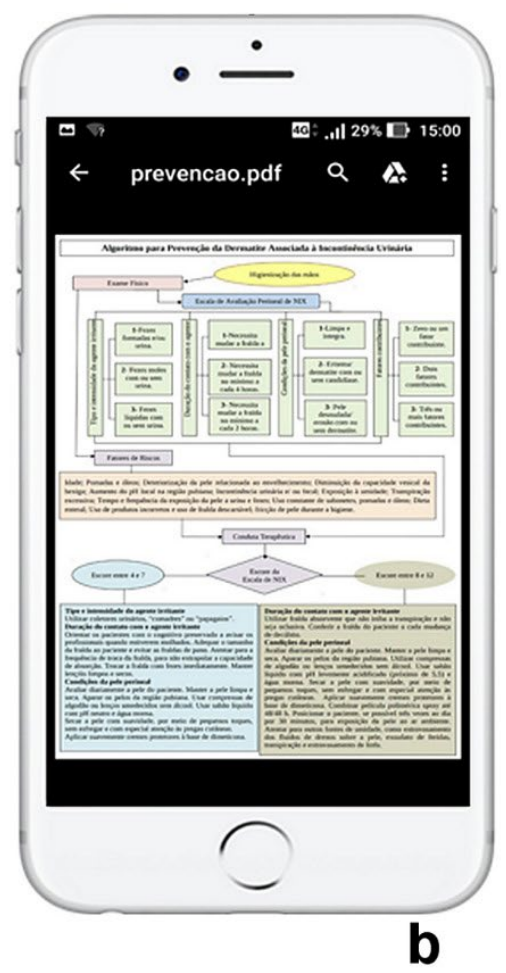

b

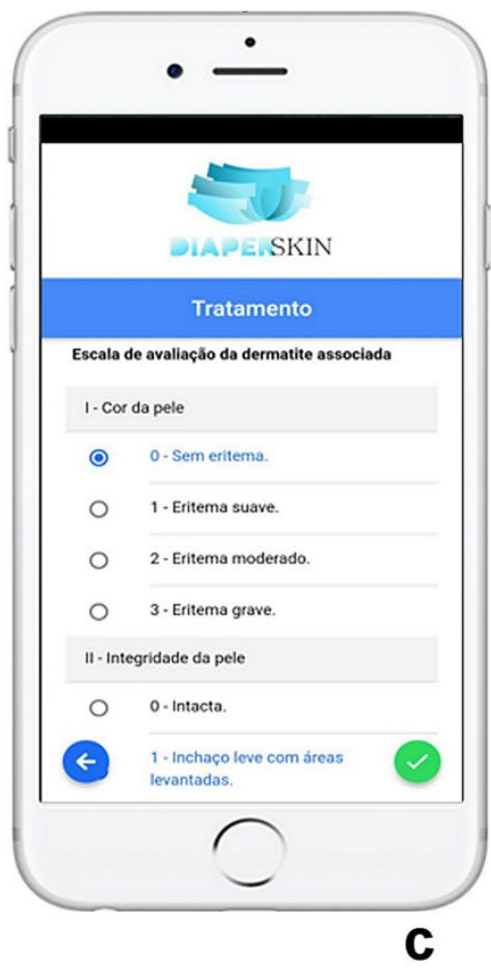

Figura 5. Exemplos de telas do aplicativo. (a) Procedimentos de avaliação e tratamento; (b) Texto parcial sobre condutas de tratamento da dermatite; (c) Algoritmo do protocolo de tratamento. Pouso Alegre, MG, Brasil, 2019

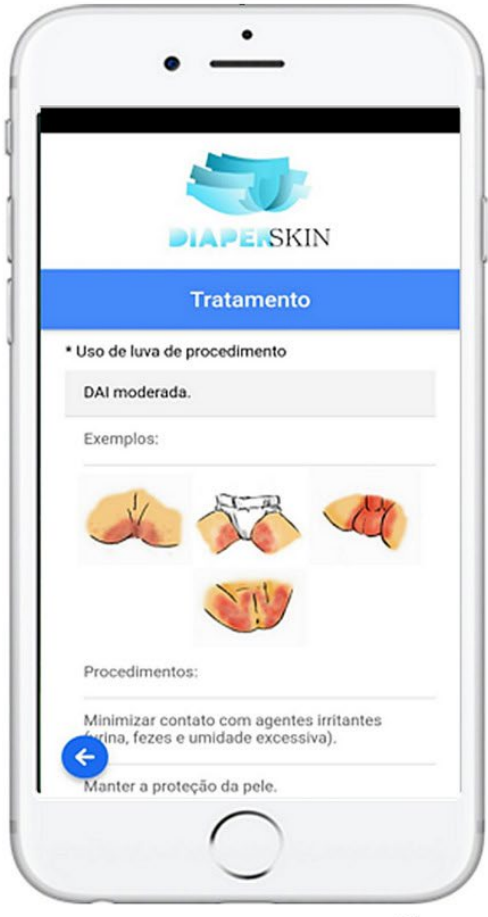

a

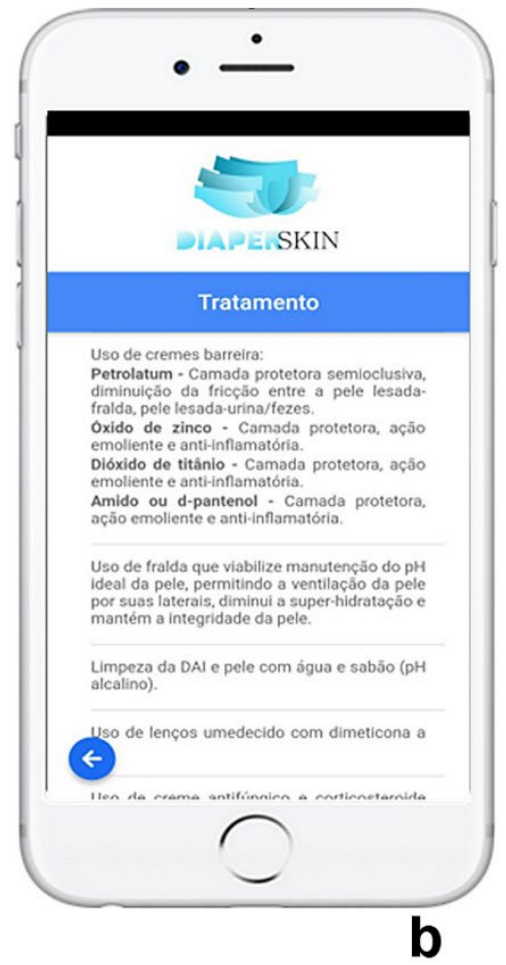


A tela inicial apresenta os nomes dos autores, o nome da instituição onde foi desenvolvido o estudo e três ícones: envelope, o qual fornece um endereço de eletrônico (e-mail) para contato, caso o usuário tenha dúvidas ou sugestões; informações, o qual abre uma tela com a definição de DAl; e opções. O ícone opções leva a uma tela onde o usuário poderá consultar as referências bibliográficas inerentes ao aplicativo ou iniciar a avaliação da pele nas regiões genital, perigenital e perineal (Figura 2a), dos fatores de risco (Figura 2b), e aplicar a escala PAT (Figura 2c). Segue-se a tela com as opções de procedimentos para prevenção ou tratamento da DAl (Figura 3a). O ícone prevenção dá acesso às condutas terapêuticas preventivas (Figuras 3b, 3c, 4a) e ao protocolo clínico em forma de algoritmo (Figura 4b), o qual está disponível para download. Após anamnese e a avaliação clínica (Figura 4c), o usuário poderá abrir as telas com as condutas terapêuticas para o tratamento da DAI (Figuras $5 a$ e 5b) e a tela com o algoritmo para o tratamento, disponível para download (Figura 5c).

\section{Discussão}

O uso de tecnologias computacionais na área educacional e da saúde vem inovando as relações ensino-aprendizagem e teoria-prática na assistência. A utilização de ambientes virtuais para a educação continuada tem demonstrado que a interatividade favorece o processo de aprendizado e o aprimoramento na assistência segura sem danos para o paciente ${ }^{15-20}$. O uso de aplicativos no meio clínico contribui para a anotação e evolução da assistência, que tem como consequência a continuação do tratamento e do cuidado com segurança ${ }^{13}$. A assistência individualizada, personalizada, prestada aos pacientes com lesão reduz o tempo de cicatrização e concorre para a análise dos custos e eficácia do tratamento utilizado ${ }^{21-22}$. Aplicativos móveis possibilitam mobilidade dos usuários, os quais podem utilizar o seu smartphone pessoal durante as 24 horas do dia e onde estiverem.

Ao avaliar o paciente com DAI, o enfermeiro precisa tomar decisões, e estas devem ser baseadas em evidências científicas, conhecimento da fisiologia e anatomia da pele. Sem estes conhecimentos, o profissional não conseguirá prescrever a cobertura ideal, avaliar e acompanhar a evolução da lesão ${ }^{20,23}$.
O aplicativo desenvolvido fornece medidas para a prevenção e tratamento da DAl, buscando a promoção da cicatrização da lesão por meio da assistência sistematizada, individualizada, com qualidade, sem riscos ou danos para o paciente. Ele pode ser utilizado como ferramenta de apoio pedagógico, teórico e prático e propicia um ambiente em que profissional ou estudantes passam por ciclos de reflexão e ação $0,7,20$.

As telas foram construídas com imagens simples, vocabulário acessível e claro, e textos curtos com informações suficientes para a compreensão do tema. 0 tipo e tamanho da fonte foram escolhidos objetivando uma estética harmoniosa e que o conteúdo fosse visível ao usuário. O texto de um aplicativo deve apresentar um vocabulário de fácil entendimento, uma leitura que promove o interesse do usuário na utilização. A escolha e a apresentação do conteúdo devem levar em consideração a sua capacidade em acionar os conhecimentos prévios dos estudantes,$\frac{7,20}{}$.

Como ferramenta principal, as figuras e fotos de um aplicativo devem ser construídas de forma clara, objetiva e, principalmente, pedagógica, de maneira a favorecer a alteração da rotina na condução do conteúdo e possibilitar vários modos de apreensão do assunto. A figura ou fotos devem motivar o usuário a promover o conhecimento intuitivo e proporcionar a compreensão de conceitos que, se fossem dispostos somente pela via textual, seriam mais difíceis de serem assimilados ${ }^{20}$.

A união de texto, figuras e fotos deve imprimir agilidade no processo de aprendizagem, oferecendo ambientes para as ações de Enfermagem, contribuindo para uma prática baseada em evidências. Esse aplicativo deve propiciar ao enfermeiro resgatar as informações contidas em suas telas e também promover o desenvolvimento do conhecimento, permitindo ao usuário definir o seu próprio caminho, o que se traduz em um enorme potencial para a educação $15-20$.

Este aplicativo foi construído pensando na importância das cores, do tamanho, tipo de letra adequada para que o usuário possa ter boa leitura e compreensão do tema e das telas relacionados ao conteúdo.

As telas de um aplicativo devem ser construídas com cores claras e fundos simples, utilizando cores neutras, porque aumentam a visibilidade das outras cores presentes no texto. A cor é um recurso relevante e pode fazer diferença em um texto, dependendo da posição e do contraste ${ }^{13-21}$. 
Caso o profissional constate que o paciente não apresenta DAl, após avaliar a pele nas regiões genital, perigenital e perineal, poderá aproveitar-se das orientações relacionadas à técnica da higiene dessas regiões, medidas preventivas e condutas terapêuticas pertinentes à dermatite.

Vários estudos relatam que, para evitar a DAl, é necessário que o profissional, paciente e cuidador utilizem fralda que absorva a umidade da pele, urinas, fezes e sempre que o paciente urinar ou evacuar, a troca da fralda deve ser imediata $3-4,20,23$.

Uma das preocupações que o profissional deverá ter é investigar se há presença de bactérias, tendo em vista que uma área da pele úmida, com existência de fezes e urina, tem como consequência a formação de bactérias que podem originar infecção secundária pela lesão cutânea instalada $a^{3-4,23}$.

Um estudo avaliou o custo na utilização da cobertura One Step Incontinence System no tratamento da incontinência fecal e urinária. Foram utilizados dois lenços umedecidos destinados à limpeza, hidratação e proteção; e o outro produto foi um absorvente (fralda). Ficou demonstrado que o OSIS contribui para a diminuição da DAl, pois este produto promove a limpeza da pele com presença de suor, urina e fezes, reduz também a permanência de fezes e urina em contato com a pele em região de fralda9, 23 .

Em relação aos produtos para tratar a DAl, a borrifação de produto-barreira à base de película de polímero nas regiões genital, perigenital e perineal, previne a DAI e tem excelente custo-benefício no tratamento desse agravo. A utilização de fraldas superabsorventes, lenços umedecidos impregnados com dimeticona 3\% e spray para a formação de película de polímero, previne a DAl e também é uma ótima opção para trata-la. O tratamento com antifúngico e corticoide é indicado nos casos de infecção 3,20,23.

A utilização de cremes-barreira contribui para prevenir a umidade na área das fraldas, diminui desaparecimento da transepidérmica de água, reduz a permeabilidade da pele, e minimiza o contato das fezes com a pele, pois os cremes aderem à epiderme.

\section{Conclusão}

Após revisão integrativa da literatura, foi desenvolvido o aplicativo "DIAPERSKIN", o qual tem grande potencial de uso na prática clínica do profissional da saúde, durante a avaliação da DAl, na escolha de medidas preventivas e do produto ideal para tratar a DAl, e para a educação continuada desses profissionais, pois o aplicativo "DIAPERSKIN" é uma tecnologia inovadora.

A limitação do estudo foi a não validação do aplicativo DIAPERSKIN, sendo essa a nossa perspectiva futura do estudo.

\section{Contribuições dos autores}

Rocha CA contribuiu na concepção do trabalho, na redação do artigo e na aprovação final da versão a ser publicada. Salomé GM contribuiu na concepção do trabalho, na interpretação dos achados e na aprovação final da versão a ser publicada.

\section{Conflitos de interesses}

Nenhum conflito financeiro, legal ou político envolvendo terceiros (governo, empresas e fundações privadas, etc.) foi declarado para nenhum aspecto do trabalho submetido (incluindo, mas não se limitando a subvenções e financiamentos, participação em conselho consultivo, desenho de estudo, preparação de manuscrito, análise estatística, etc.).

\section{Referências}

1. Mittag BF, Krause TC, Roehrs H, Meier MJ, Danski MT. Care of Skin Injuries: Nursing Actions. Rev Estima. 2017;15(1):19-25. https://doi.org/10.5327/Z1806-3144201700010004

\section{Massahud Jr MR, Salomé GM, Ferreira LM. Low intensity laser and microcorrent in scratching of skin wounds in rats. J Nurs UFPE Online. 2017;11(Suppl. 9):3567-73. http://dx.doi.org/10.5205/ reuol.10620-94529-1-SM.1109sup201709}

3. Gray M. Context for practice: prevention of pressure injury and incontinence-associated dermatitis. J Wound Ostomy Continence Nurs. 2017;44(5):406-8. http://dx.doi.org/10.1097/ WON.0000000000000367 
4. Gray M, Giuliano KK. Incontinence-associated dermatitis, characteristics and relationship to pressure injury: a multisite epidemiologic analysis. J Wound Ostomy Continence Nurs. 2018;45(1):63-7. http://dx.doi.org/10.1097/ WON.0000000000000390

5. Gray M, Bliss DZ, Doughty DB, Ermer-Seltun J, Kennedy-Evans $\mathrm{KL}$, Palmer $\mathrm{MH}$. Incontinence-associated dermatitis: a consensus. J Wound Ostomy Continence Nurs. 2007;34(1):45-54. http://dx.doi. org/10.1097/00152192-200701000-00008

6. Cunha DR, Salomé GM, Massahud Jr MR, Mendes B, Ferreira LM. Development and validation of an algorithm for laser application in wound treatment. Rev. Latino-Am. Enfermagem. 2017;25:e2955. http://dx.doi.org/10.1590/1518-8345.1998.2955

7. Carvalho MRF, Salomé GM, Ferreira LM. Construction and validation of algorithm for treatment of pressure injury. J Nurs UFPE online. 2017;11(Suppl. 10):4171-83. http://dx.doi. org/10.5205/reuol.10712-95194-3-SM.1110sup201722

8. Santos AC, Dutra RAA, Salomé GM, Ferreira LM. Construction and internal reliability of an algorithm for choice cleaning and topical therapy on wounds. J Nurs UFPE Online.

2018;12(5):680-5. http://dx.doi.org/10.5205/1981-8963v12i5a230675p1250-1262-2018

9. Jordan J. Prevention of incontinence-associated dermatitis in people with dementia. J 10-Neurosci Nurs. 2015;11(5):228-34. http://dx.doi.org/10.12968/bjnn.2015.11.5.228

10. Malaquias SG, Bachion MM, Nakatani AYK. Risco de integridade da pele prejudicada em idosos hospitalizados. Cogitare Enferm. 2008; 13(3):428-36. http://dx.doi.org/10.5380/ ce.v13i3.13040

11. Marin HF. Health information system: general considerations. J Health Inform. [Internet]. 2010;2(1):20-4. Disponível em: http://www.jhi-sbis.saude.ws/ojs-jhi/index.php/jhi-sbis/article/ viewFile/4/52

12. Vêscovi SJ, Primo CC, Sant'Anna HC, Bringuete MEO, Rohr RV, Prado TN, et al. Mobile application for evaluation of feet in people with diabetes mellitus. Acta Paul Enferm. 2017;30(6):607-13. http://dx.doi.org/10.1590/1982-0194201700087

13. Salomé GM, Bueno JC, Ferreira LM. Multimedia application in a mobile platform for wound treatment using herbal and medicinal plants. J Nurs UFPE Online. 2017;(Suppl. 11):4579-88. http:// dx.doi.org/10.5205/1981-8963-v11i11a231197p4579-4588-2017
14. Salomé GM, Alves NF. Developing a mobile app for prevention and treatment of pressure injuries. Adv Skin Wound Care. 2018;31(2):1-6. http://dx.doi.org/10.1097/01. ASW.0000529693.60680.5e

15. Alves NF, Salomé GM. App “SICKSEG" in mobile platforms for the prevention of skin injuries. J Nurse UFPE on line. 2020;14:e244152. http://dx.doi.org/10.5205/19818963.2020 .244152

16. Arnold-Long M, Johnson Ey. Epidemiology of IncontinenceAssociated Dermatitis and Intertriginous Dermatitis (Intertrigo) in an Acute Care Facility. J Wound Ostomy Continence Nurs. 2019;46(3):201-206. http://dx.doi.org/10.1097/ WON.0000000000000519

17. Holanda VR, Pinheiro AKB. Development of a hypermedia system for interactive teaching of sexually transmitted diseases. J Nurs UFPE Online. 2015;9(Suppl 2):781-9. http://dx.doi. org/10.5205/reuol.6391-62431-2-ED.0902supl201502

18. Barra DCC, Paim SMS, Sasso GTMD, Colla GW. Métodos Para Desenvolvimento De Aplicativos Móveis Em Saúde: Revisão Integrativa Da Literatura. Texto Contexto Enferm, 2017;26(4):e2260017. http://dx.doi.org/10.1590/010407072017002260017

19. Brandão ACMAG, Gambin CC, Majado CA, Kunitake N, Alexandre NMC, Dantas SRPE. Adaptação do instrumento "Perineal Assessment Tool" para a cultura brasileira. ESTIMA Braz J Enterostomal Ther. 2018. 16:e0618. http://dx.doi.org/10.30886/ estima.v16.397_PT97_PT

20. Qiang L, Xian LW, Bin PY, Hang SM. Investigating ICU nurses' understanding of incontinence-associated dermatitis: an analysis of influencing factors. WCET ${ }^{\circledR}$ Journal. [Internet]. 2020;40(1):32-38. Disponível em: https://search.informit.com.au/ documentSummary;dn=064116072725918; res=IELHEA

21. Pereira FGF, Silva DV, Sousa LMO, Frota NM. Building a digital application for teaching vital signs. Rev Gaucha Enferm. 2016;37(2):e59015. http://dx.doi.org/10.1590/1983$\underline{1447.2016 .02 .59015}$

22. Galvão ECF, Puschel VAA. Aplicativo multimídia em plataforma móvel para o ensino da mensuração da pressão venosa central. Rev Esc. Enferm USP.2012; 46(Esp):107-15. http://dx.doi. org/10.1590/S0080-62342012000700016

23. Salomé GM, Rocha CA, Miranda FD, Alves JR, Dutra RAA, Tenório AG. Algoritmos para prevenção e tratamento de dermatite associada à incontinência. ESTIMA Braz J Enterostomal Ther. 2020;18:e1320. http://dx.doi.org/10.30886/estima.v18.837 PT 